\title{
The preventive effect of aldose reductase inhibition on diabetic optic neuropathy in the $\mathrm{BB} / \mathrm{W}$-rat
}

\author{
M. Kamijo, P. V.Cherian, A. A. F. Sima \\ Departments of Pathology and Internal Medicine, and the Michigan Diabetes Research and Training Center, \\ University of Michigan Medical Center, Ann Arbor, Michigan, USA
}

Summary. A polyol-pathway-related mechanism has been invoked in the pathogenesis of murine and human diabetic peripheral neuropathy in which progressive axonal atrophy and axo-glial dysjunction constitute the cardinal structural abnormalities. We have previously reported similar neuroanatomical changes in the optic nerve of 6-month diabetic $\mathrm{BB} / \mathrm{W}$-rats. In the present study we demonstrate progression of axonal atrophy and axo-glial dysjunction in the optic nerve in 12-month diabetic BB/W-rats. These structural lesions showed highly significant correlations with the associated prolongation of the latencies of the visual evoked potentials, suggesting that axo-glial dysjunction and axonal atrophy are major determinants for impaired optic nerve function. As in peripheral nerve, the polyol-pathway is present in the optic nerve and is activated by hyperglycaemia and galactosaemia. In this study we further examined the treatment effect of the aldose reductase inhibitor ponalrestat, given from 3 weeks of diabetes and continued throughout the study protocol. This regimen resulted in complete prevention of axo-glial dysjunction, and had a significant ameliorating effect on visual evoked potential latencies, but had no effect on optic nerve axonal atrophy. This latter finding differs from the effect of aldose reductase inhibition on diabetic peripheral nerve and suggests that axonal atrophy of central nerve tracts in diabetes may be the consequence of other metabolic abnormalities or alternatively the present regimen was insufficient to protect central axons from the effects of an increased activity of the polyol pathway.

Key words: Optic neuropathy, visual evoked potential, diabetic $\mathrm{BB} / \mathrm{W}$-rat, aldose reductase inhibition.
Neuropathy is the most common symptomatic complication affecting patients with diabetes and encompasses a spectrum of abnormalities involving peripheral somatic and autonomic nerve function [1]. The pathophysiology of diabetic neuropathies has been studied extensively in the spontaneously diabetic BB/W-rats, which has served as a model of human Type 1 (insulin-dependent) diabetes mellitus [2-7]. Experimental studies in this and other animal models, and in humans have implicated an activation of the polyol pathway as the initiating metabolic abnormality underlying impaired nerve function and subsequent developments of nerve fibre atrophy, injury and loss $[1,8,9]$. The increased flux of excess glucose through the polyol pathway is mediated by the enzyme aldose reductase and results in intracellular accumulation of sorbitol and a compensatory decrease in myo-inositol content, the major consequences of which are defective neural $\mathrm{Na}^{+} / \mathrm{K}^{+}$ATPase and decreased nerve function [8-12].

In diabetic animal models the acute nerve dysfunction is reversed by the treatment with aldose reductase inhibitors (ARI's), which also prevent the long-term neuroana- tomical abnormalities from occurring $[13,14]$. In diabetic patients with established and symptomatic peripheral neuropathy, experimental ARI's improve nerve function as a consequence of increased nerve fibre regeneration and repair of nodal and axonal structural injuries [15-17].

The possible effects of hyperglycaemia on central nervous system sensory tracts have not previously been subjected to experimental scrutiny, although functional and neuropsychological impairments in diabetic patients suggest that the central nervous system is not spared from the complications of diabetes [18-22]. In a recent study we demonstrated optic nerve fibre atrophy and nodal injury accompanied by increased visual evoked potential (VEP) latencies in 6-month diabetic BB/W-rats [23]. We suggested that this central sensory neuropathy might result from an activated polyol-pathway, in keeping with the demonstration of elevated sorbitol levels in the optic nerve of diabetic and galactosaemic rats [24; unpublished observations]. In the present study the earlier experiments were extended, and we now report on the progression of diabetic optic neuropathy in 12-month diabetic 
Table 1. Data obtained at 12 months of diabetes in $\mathrm{BB} / \mathrm{W}$ rats

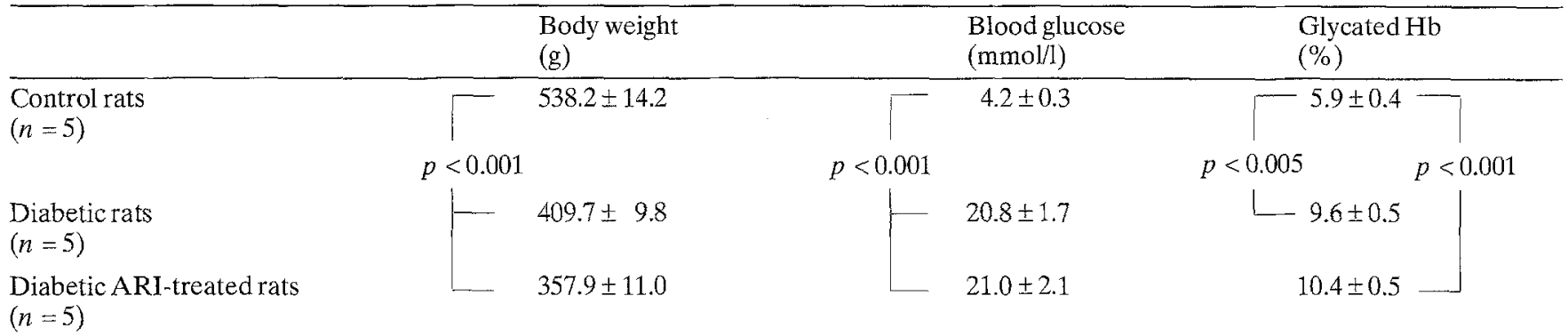

$\mathrm{BB} / \mathrm{W}$-rats, and its partial prevention by an ARI. The current results are compared with previously reported findings obtained at 6 months of diabetes, and the functional impairments reflected by the VEP are correlated with specific neuroanatomical lesions.

\section{Materials and methods}

\section{Animals}

Pre-diabetic male $\mathrm{BB} / \mathrm{W}$-rats and non-diabetes-prone male $\mathrm{BB} / \mathrm{W}$ rats were obtained from the National Institutes of Health sponsored $\mathrm{BB} / \mathrm{W}$-rat colony at the University of Massachusetts (Worcester, Mass., USA). Daily urine glucose monitoring was performed to ascertain the onset of diabetes.

Following detection of diabetes, BB/W-rats were supplemented with daily doses of protamine zinc insulin $(0.5-3.0 \mathrm{IU} /$ day) (Novo Nordisk, Copenhagen, Denmark) in order to prevent ketoacidosis and to keep diabetic animals hyperglyeaemic for 12 months. At 3 weeks of diabetes, one group of diabetic animals were given the ARI ponalrestat (ICl-Pharma, Macclesfield, Cheshire, UK) in the rat chow, calculated so that a minimum of $20 \mathrm{mg}$ ponalrestat $/ \mathrm{kg}$ body weight was administered per day [13]. This regimen was continued throughout the study protocol. Animals were kept individually in metabolic cages with free access to rat chow (Wayne Lab Blox F-6; Wayne Laboratory Animal Diets, Chicago, III., USA) and water, in a room with a 12-h light/dark cycle. Urine was monitored on a daily basis with respect to volume, glucose and ketone contents (Test Tape; Eli Lilly, St. Louis, Mo., USA), and insulin doses were titrated accordingly as previously described [23]. Body weights and blood glucose levels were measured biweekly (Glucometer; Miles, Ames Division, Elkhart, Ind., USA) and glycated haemoglobin levels were obtained every month (Glycotest; Pierce Chemical Co., Rockford, IIl., USA). Diabetic animals were examined for the presence of cataract.

\section{Visual evoked potential}

Animals were dark-adapted for at least $12 \mathrm{~h}$ prior to the VEP recordings. They were sedated with a mixture of ketamine hydrochloride (100 $\mathrm{mg} / \mathrm{kg}$ body weight) and aceproniazine moleate $(25 \mathrm{mg} / \mathrm{kg}$ body weight) injected i.p. Pupils were dilated with $1 \%$ tropicamide and the cornea was anaesthetised with $0.5 \%$ proparacain hydrochloride. VEP's were recorded from the occipital crest in the midline $(\mathrm{Oz})$, and were referenced to the midline of the forehead $(\mathrm{Fz})$ using platinum subdermal electrodes (Grass Instrument Co., Quincy, Mass., USA) as previously described in detail [23]. Recordings were averaged 16 times, and recorded with an electro-retino-gram (ERG) recorder (Life-Tech Instruments, Inc., Houston, Tx., USA) on chart paper with pass filters set at 0.1 and $500 \mathrm{~Hz}$. Light stimuli were delivered at an intensity of $4.82 \times 10^{-3} \mathrm{lumen} \mathrm{s}^{-1} \mathrm{~cm}^{-1}$ at a frequency of
$2 \mathrm{~Hz}$ and a duration of $1 \mu$ s by an ERG stimulator (Life Tech Instruments, Inc.). The light source was fitted with a diffuser and was placed $0.5 \mathrm{~m}$ from the head of the rat [23].

\section{Tissue collection}

After 12 month of diabetes, ARI-treated and non-ARI-treated diabetic animals, and age-matched non-diabetic control rats were anaesthetised with $50 \mathrm{mg}$ sodium phenobarbital/kg body weight and killed by whole body perfusion with a $0.1 \mathrm{~mol}$ cacodylate buffered (pH 7.40) $2.5 \%$ glutaraldehyde solution. The intracranial portion of the right optic nerve was sampled and post-fixed in cacodylate buffered ( $\mathrm{pH} \mathrm{7.40)} 1 \%$ osmium tetroxide for $2 \mathrm{~h}$ in $4^{\circ} \mathrm{C}$, dehydrated in increasing concentrations of alcohol, embedded in Epon, and examined electron microscopically.

\section{Morphometric analysis}

Electron micrographs with a total magnification of $7,000 \times$ were used to measure the cross-sectional areas of 1,000 systematic randomly chosen myelinated optic nerve fibres. The areas of the corresponding neuropil were measured in each optic nerve. These measurements were used to calculate mean myelinated fibre size, fibre density and occupancy as described in detail previously [23]. In addition, from the same 1,000 nerve fibres per optic nerve the axonal size alone was measured and expressed as mean axonal size. From longitudinally sectioned optic nerves the frequency of axo-glial dysjunction was calculated from $11.4 \pm 0.7$ nodes of Ranvier obtained from serial sections of each optic nerve. Axo-glial dysjunction is defined as the frequency of myelin loops devoid of axo-glial junctions. Only sections in which both the terminal myelin loops and the opposing axolemma displayed clearly discernable trilaminar membranes were included in this morphometric analysis as previously described [6, 23, 25].

\section{Statistical analysis}

Results are expressed as mean \pm SEM and the significance of differences between groups was calculated by the ANOVA test and Wilcoxon's non-parametric $t$-test. Regression analyses were performed using the least square method.

\section{Results}

\section{Body weights and hyperglycaemia}

After 12 months of diabetes, non-ARI-treated and ARItreated diabetic rats showed a $24 \%$ and $34 \%$ reduction in body weight gain, respectively, when compared to age- 
Table 2. Latencies $\left(\mathrm{P}_{1} ; \mathrm{P}_{2} ; \mathrm{N}_{1} ; \mathrm{N}_{2}\right)$ of visual evoked potentials in control, diabetic and ARI-treated diabetic rats at 1 year of diabetes

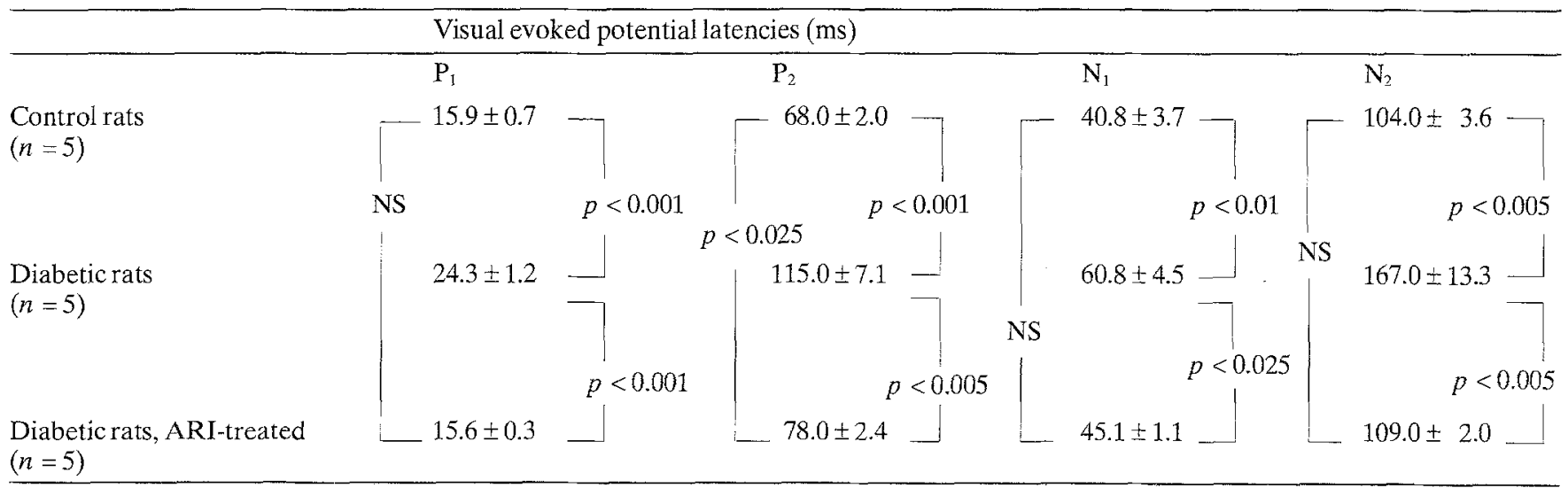

Table 3. Myelinated fibre morphometry in control, diabetic and ARI-treated diabetic BB/W-rats

\begin{tabular}{|c|c|c|c|c|c|}
\hline & $\begin{array}{l}\text { Myelinated } \\
\text { fibre size }\left(\mu \mathrm{m}^{2}\right)\end{array}$ & $\begin{array}{l}\text { Axonal size } \\
\left(\mu \mathrm{m}^{2}\right)\end{array}$ & $\begin{array}{l}\text { Myelinated fibre } \\
\text { density }\left(\# / \mathrm{mm}^{2}\right)\end{array}$ & $\begin{array}{l}\text { Myelinated } \\
\text { fibre occupancy }\end{array}$ & $\begin{array}{l}\text { Axo-glial } \\
\text { dysjunction }(\%)\end{array}$ \\
\hline $\begin{array}{l}\text { Control rats } \\
(n=5)\end{array}$ & $1.81 \pm 0.05$ & $0.73 \pm 0.03$ & $384,845 \pm 11,845$ & $69.5 \pm 1.8$ & $\begin{array}{c}1.99 \pm 0.87 \\
\mid \\
p<0.01\end{array}$ \\
\hline $\begin{array}{l}\text { Diabetic rats } \\
(n=5)\end{array}$ & $1.62 \pm 0.12$ & $0.54 \pm 0.06$ & $449,954 \pm 54,481$ & $70.1 \pm 2.2$ & $\begin{array}{c}5.94 \pm 1.39 \\
\mid \\
p<0.01\end{array}$ \\
\hline
\end{tabular}

matched non-diabetic control rats. Elevated blood glucose and glycated haemoglobin levels were not affected by ARI-treatment in diabetic rats (Table 1 ). Dense cataracts were not observed in any of the diabetic animals.

\section{Visual evoked potentials}

Chronic non-ARI-treated diabetic BB/W-rats showed significantly prolonged latencies of both the positive $\left(P_{1}\right.$ and $\mathrm{P}_{2}$ ) and the negative $\left(\mathrm{N}_{1}\right.$ and $\mathrm{N}_{2}$ ) deflections of the VEP indicating a slowed nerve conduction velocity of the central optic nerve tract. The prolongation of the latencies varied between $33 \%$ and $42 \%$ (Table 2 ). ARI treatment of diabetic rats for 1 year resulted in an almost complete prevention of the optic system nerve conduction velocity, as indicated by the latencies of the four deflections of the VEP (Table 2). Only the $\mathrm{P}_{2}$ latency showed a significant $(p<0.025) 13 \%$ residual defect (Table 2$)$. The amplitudes of the VEP's were not affected by diabetes or by ARI treatment (data not shown).

\section{Morphometric changes}

Myelinated optic nerve fibres in non-ARI-treated diabetic rats showed a $10 \%$ non-significant atrophy when compared to age-matched non-diabetic control rats (Table 3). ARI-treatment of diabetic rats had no effect on mean myelinated fibre size (Table 3). When axonal size alone was assessed, non-ARI-treated diabetic rats demonstrated a $28 \%$ significant $(p<0.05)$ reduction in axonal size. However, this axonal atrophy of optic nerve fibres was not prevented by ARI-treatment (Table 3). Myelinated fibre density and myelinated fibre occupancy were not altered in non-ARI-treated or ARI-treated diabetic rats when compared with non-diabetic control rats (Table 3).

Axo-glial dysjunction, a characteristic abnormality of diabetic peripheral neuropathy, was increased three-fold $(p<0.001)$ in optic nerve fibres of 12 -month diabetic $\mathrm{BB} / \mathrm{W}$-rats. ARI-treatment, which had no effect on hyperglycaemia, was associated with a complete prevention of optic nerve axo-glial dysjunction (Table 3).

\section{Correlations between axo-glial dysjunction, myelinated fibre size, and VEP}

In a previous study [23] we reported differences in myelinated optic nerve fibre morphometry between 6-month diabetic BB/W-rats and age-matched non-diabetic control rats. The methods used in the previous study were identical to those employed in the current study. Therefore, for the purpose of structural-functional correlations, the previously reported data were combined with the present results. The $\mathrm{P}_{2}$ deflection of the VEP is believed to represent one of the two optic nerve action potentials, whereas $\mathbf{N}_{1}$ 


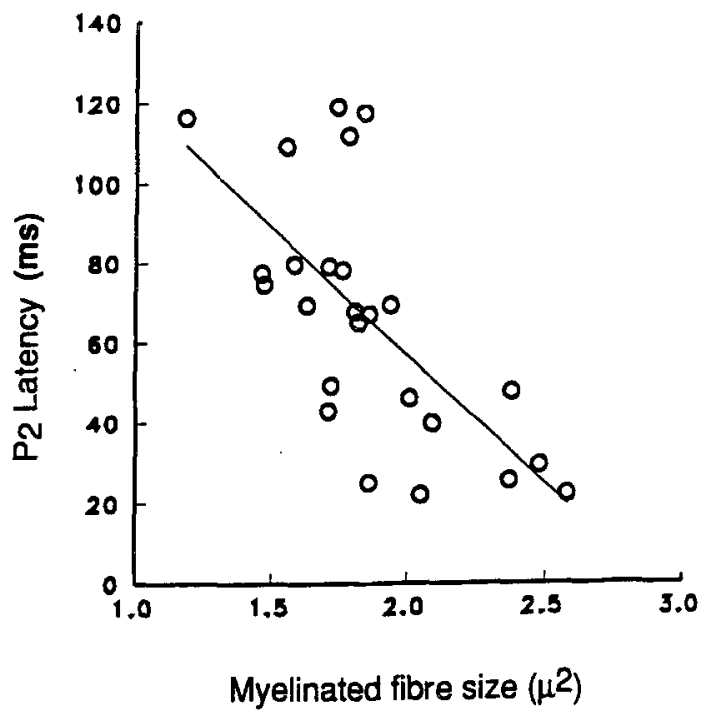

Fig. 1. Linear regression with myelinated fibre size as the independent and $\mathrm{P}_{2}$ latency as dependent variables. The data points represent 6-month control and diabetic rats [20], and 12-month control, diabetic and ARI-treated diabetic rats. $r^{2}=0.46 ; p=0.0002$

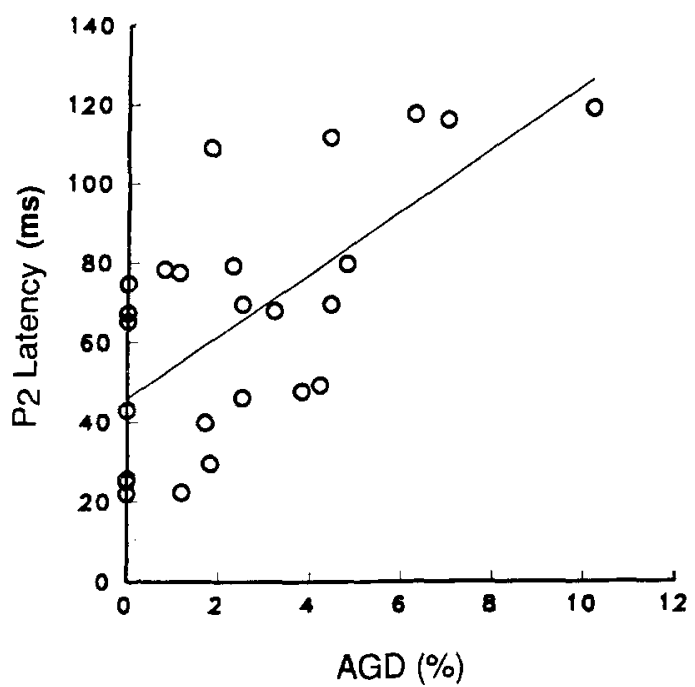

Fig. 2. The $\mathrm{P}_{2}$ latency correlated closely with axoglial dysjunction (AGD) in 6-month control and diabetic rats, and 12-month control, diabetic, and ARI-treated diabetic rats. $r^{2}=0.43 ; p=0.0003$

and $\mathrm{N}_{2}$ represent the compound post-synaptic responses of the geniculo-calcarine tract [26]. The $\mathrm{P}_{2}$ latency was therefore used for the correlations with the structural abnormalities of the optic nerve.

Linear regression analysis between myelinated fibre size and the latency of $\mathrm{P}_{2}$ in 6-month diabetic and control rats [23] and 12-month diabetic, ARI-treated diabetic and control rats revealed a highly significant negative correlation $\left(y=-64.03 x+185.02 ; n=25 ; r^{2}=0.46 ; p=0.0002\right)$ (Fig.1) suggesting that myelinated fibre size is a major determinant for the conduction velocity in the optic nerve. However, when axo-glial dysjunction was used as the independent variable and regressed against the latency of $\mathrm{P}_{2}$ a similar but positive correlation was obtained $\left(y=7.88 x+46.1 ; n=25 ; r^{2}=0.43 ; p=0.0003\right)($ Fig. 2$)$ in-

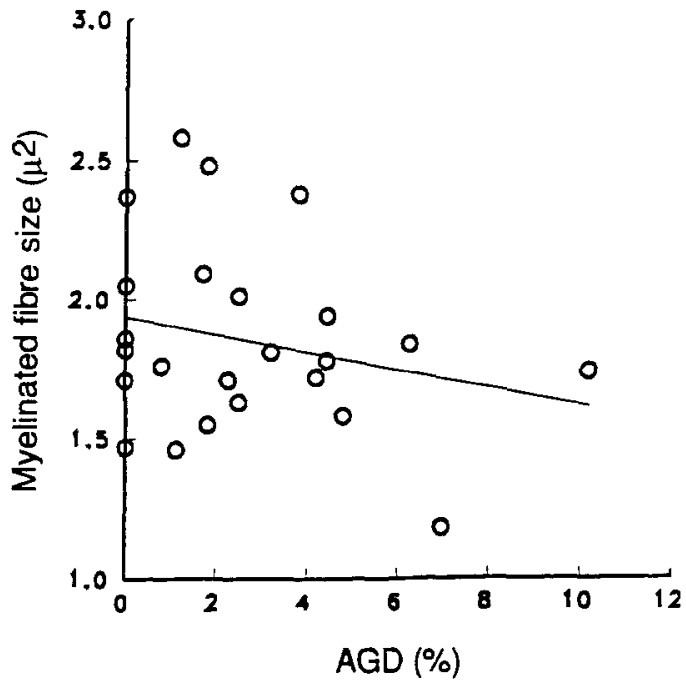

Fig. 3. No correlation was obtained between axoglial dysjunction (AGD) and myelinated fibre size in 6-month control and diabetic, and 12-month control, diabetic, and ARI-treated diabetic rats. $r^{2}=0.06 ; p=0.232$

dicating that axo-glial dysjunction is another important structural lesion responsible for an equally large part of the slowed optic nerve conduction velocity. However, the product of axo-glial dysjunction and axonal atrophy did not reveal a synergistic effect on the $\mathrm{P}_{2}$ latency $(n=25$; $r^{2}=0.46 ; p=0.0002$ ). No correlation was obtained between myelinated fibre size and axo-glial dysjunction. $\left(y=-0.03 x+1.94 ; n=25 ; r^{2}=0.06 ; p=0.232\right)$ (Fig. 3).

\section{Discussion}

Axonal atrophy and axo-glial dysjunction appear to be the two cardinal structural lesions which occur in diabetic peripheral nerve in experimental animals $[6,13]$ and in humans [27], in whom these lesions form the structural basis for the characteristic nerve conduction slowing $[13,16]$. In the present study we have demonstrated similar but less severe changes in a central sensory nerve tract, suggesting that the central nervous system is not spared from the adverse effects of diabetic dysmetabolism and that the same pathogenetic mechanisms as those we have proposed for peripheral diabetic neuropathy [28] may underlie the neuroanatomical changes which occur in the optic nerve. Axo-glial junctions provide a paranodal barrier-system that separates the voltage-sensitive nodal sodium-channels from the paranodal potassium-channels. The breach in this barrier system caused by axo-glial dysjunction is associated with diminished nodal sodium permeability and increased potassium leakage currents, resulting in a delay in the build-up of the nodal exitation potential $[5,29]$. In the extreme situation, the threshold potential is not reached rendering the node non-exitable with subsequent conduction block $[5,29,30]$. After 1 year of diabetes the myelinated fibres of the optic nerve showed a three-fold increase in axo-glial dysjunction compared to agematched control animals. There was a doubling in its fre- 
quency compared with 6-month diabetic animals [23], indicating a slow but progressive increase in the severity of this structural lesion with duration of diabetes, which is analogous to the situation in diabetic peripheral nerve of both the BB/W-rat [13] and humans [27]. This characteristic diabetic nodal lesion was completely prevented with the ARI ponalrestat, which parallels the effect of the same compound on peripheral nerve axo-glial dysjunction in the $\mathrm{BB} / \mathrm{W}$-rat $[6,13]$. Clinically overt neuropathy in diabetic patients treated with ARI, demonstrates a normalization of axo-glial dysjunction $[16,17]$, suggesting that this structural lesion is polyol-pathway dependent.

In contrast, the progressive central axonal atrophy, which increased by $20 \%$ from 6 months to 12 months of diabetes, appeared to be non-responsive to ARI-treatment. This is contrary to the situation in peripheral nerve where ARI has a significant preventive effect on axonal atrophy in the spontaneously diabetic BB/W-rat [13]. It is of interest to note, when comparing the control data on myelinated fibre size at 6 months [23] with that at 12 months, that a substantial and apparently age-related myelinated fibre atrophy has taken place, which was associated with a $64 \%$ increase in the latency of $\mathrm{P}_{2}$ in nondiabetic control rats. The latencies of the optic nerve $P_{1}$ potential and the two geniculo-calcarine potentials $\left(\mathrm{N}_{1}\right.$ and $\mathrm{N}_{2}$ ) were also prolonged in 12-month control rats compared with 6 months, but to a lesser extent (17-27\%) than that of $\mathrm{P}_{2}$. Axo-glial dysjunction showed an age-related increase from $0.60 \%$ at 6 months to $1.99 \%$ in 12 month control rats. The 1.8-fold increase in the $\mathrm{P}_{2}$ latency in diabetic rats at 6 months showed further 2.6-fold increase at 12 months of diabetes. Following 12 months of ARI-treatment the $\mathrm{P}_{2}$ latency was only increased by $13 \%$ compared to non-diabetic control rats, signifying a substantial prevention.

The present data would therefore suggest that both axo-glial dysjunction and axonal atrophy are responsible in a major way for the prolongation of the latency of the optic nerve response. However, they appear to affect the latency independently. This notion is supported by the lack of a correlation between myelinated fibre size and axo-glial dysjunction and may suggest that the mechanisms responsible for axo-glial dysjunction and myelinated fibre atrophy in central nerve tracts are different, as suggested by the divergent response to ARI-treatment. Alternatively axonal atrophy, presumably an effect of impaired protein synthesis and axonal transport [31-33], may be more sensitive to the insult inflicted by the activation of the polyol pathway, and therefore the present treatment regimen may not have been sufficient to completely inhibit the effect of the polyol pathway on axonal structural integrity.

In contrast to $P_{2}$ the latencies of $P_{1}, N_{1}$, and $N_{2}$ were completely prevented by ARI-treatment, suggesting by inference that different fibre systems, such as large myelinated fibres in the optic nerve $\left(\mathrm{P}_{1}\right)$, and the geniculo-calcarine axons $\left(\mathrm{N}_{1}\right.$ and $\left.\mathrm{N}_{2}\right)$ are less sensitive to the underlying metabolic insult than are small myelinated fibres in the optic nerve believed to conduct the $P_{2}$ action potential [26]. This notion is partly supported by the data in our previous study [23], demonstrating a more severe axonal atrophy of small myelinated fibres in the diabetic optic nerve than in large myelinated fibres. It is therefore possible that the poor response of axonal atrophy to ARItreatment may be mainly accounted for by a non-responsiveness of small myelinated axons as suggested by the VEP. The age-related changes in the VEP latencies noted between 6 and 12 months in control rats also appear to affect $P_{2}$ to a greater extent than $P_{1}, N_{1}$, and $N_{2}$. Similar findings have been reported in experimental undernutrition following which small myelinated optic nerve axons are more severely affected than large [34], and do not appear to recover electrophysiologically following nutritional rehabilitation $[35,36]$.

In summary therefore, we have demonstrated a progression of optic neuropathy in the diabetic BB/W-rat from 6 to 12 months of diabetes. The neuropathy is structurally characterized by axo-glial dysjunction and myelinated axonal atrophy and therefore similar to the structural hallmarks of diabetic peripheral neuropathy. In this central sensory neuropathy axo-glial dysjunction is completely prevented by treatment with an ARI, whereas axonal atrophy is not. This differs from the response by diabetic peripheral nerve to ARI treatment, and may suggest that mechanisms other than polyol-pathway activation are involved in the atrophy of central axons in diabetes.

Acknowledgements. This study was supported by grants from ICI Pharmaceuticals RDN-3, UK, and NIH RO1 DK43884. The authors are indebted to Ms. E. Moore for preparing this manuscript. The manuscript was presented in part at the American Diabetes Association Annual Meeting in San Antonio, Texas, June 1992.

\section{References}

1. Greene DA, Sima AAF, Albers JW, Pfeifer M (1989) Diabetic neuropathy. In: Rifkin H, Porte D (eds) Ellenberg and Rifkin diabetes mellitus, Elsevier Publishing Company, New York, pp 710-755

2. Marliss EB, Nakhooda AF, Poussier P, Sima AAF (1982) The diabetic syndrome of the BB-Wistar rat. Possible relevance to type 1 (insulin-dependent) diabetes in man. Diabetologia 22: 225-232

3. Sima AAF, Bouchier M, Christensen H (1983) Axonal atrophy in sensory nerves of the diabetic BB-Wistar rat, a possible early correlate of human diabetic neuropathy. Ann Neurol 13:264-272

4. Sima AAF (1985) Can the BB-rat help to unravel diabetic neuropathy? Annotation. Neuropath Appl Neurobiology 11: 253-264.

5. Brismar T, Sima AAF (1981) Changes in nodal function in nerve fibres of the spontaneously diabetic BB-Wistar rat. Potential clamp analysis. Acta Physiol Scand 113: 499-506

6. Sima AAF, Lattimer SA, Yagihashi S, Greene DA (1986) “Axoglial dysjunction": a novel structural lesion that accounts for poorly reversible slowing of nerve conduction in the spontaneously diabetic BB-rat. J Clin Invest 77: 474-484

7. Yagihashi S, Sima AAF (1986) Neuroaxonal and dendritic dystrophy in diabetic autonomic neuropathy. J Neuropath Exp Neurol 45: 545-565

8. Sima AAF, Stevens MJ, Feldman EL, Cherian PV, Greene DA (1993) Animal models as tools for the testing of preventive and therapeutic measures in diabetic neuropathy. In: Shafrir E (ed) Lessons from animal diabetes IV. Smith Gordon, London, pp 177-191

9. Greene DA, Lattimer SA, Sima AAF (1987) Sorbitol, myo-inositol and sodium-potassium ATPase in the pathogenesis of diabetic complications. N Engl J Med 316: 599-606 
10. Gi ne DA, Chakrabarti S, Lattimer SA, Sima AAF (1987) Role of sorbitol accumulation and myoinositol depletion in paranodal swelling of large myelinated nerve fibres in the insulindeficient spontaneously diabetic biobreeding rat. J Clin Invest 79: $1479-1485$

11. Bagnasco SM, Murphy HR, Bedfored JJ, Burg MB (1988) Osmoregulation by slow changes in aldose reductase and rapid changes in sorbitol flux. Am J Physiol 254: C788-C792

12. Stevens MJ, Lattimer SA, Kamijo M et al. (1993) Osmotically induced nerve taurine depletion and the compatible osmolyte hypothesis in experimental diabetic neuropathy in the rat. Diabetologia 36: 608-614

13. Sima AAF, Prashar A, Zhang W-X, Chakrabarti S, Greene DA (1990) Preventive effect of long term aldose reductase inhibition (Ponalrestat) on nerve conduction and sural nerve structure in the spontaneously diabetic BB-rat. J Clin Invest 85: 1410-1420

14. Yagihashi S, Kamijo M, Ido Y, Mirrlees DJ (1990) Effects of long-term aldose reductase inhibition on development of experimental diabetic neuropathy: ultrastructural and morphometric studies of sural nerve in streptozotocin induced diabetic rats. Diabetes 39: 690-696

15. Sima AAF, Bril V, Nathaniel V et al. (1988) Regeneration and repair of myelinated fibres in sural nerve biopsies from patients with diabetic neuropathy treated with an aldose reductase inhibitor. N Engl J Med 319: 548-555

16. Sima AAF, Prashar A, Nathaniel V et al. (1993) Overt diabetic neuropathy: repair of axo-glial dysjunction and axonal atrophy by aldose reductase inhibition and its correlation to improvement in nerve conduction velocity. Diabetic Med 10: 115-121

17. Sima AAF, Greene DA, Brown MB et al. (1993) Effect of hyperglycaemia and the aldose reductase inhibitor tolrestat on sural nerve biochemistry and morphometry in advanced diabetic peripheral polyneuropathy. J Diab Compl 7: 157-169

18. Ymazaki H, Adachi-Usami E, Chiba J (1982) Contrast thresholds of diabetic patients determined by VECP and psychological measurements. Acta Ophthalmol 60: 386-392

19. Bresnick GH (1986) Diabetic retinopathy viewed as a neurosensory disorder. Arch Ophthalmol 104: 989-990

20. Algan M, Ziegler O, Gehin PH et al. (1989) Visual evoked potentials in diabetic patients. Diabetes Care 12: 227-229

21. Skenazy JA, Bigler ED (1984) Neuropsychological findings in diabetes mellitus. J Clin Psychol 40:246-258

22. DeJong RN (1977) CNS manifestations of diabetes mellitus. Postgrad Med 61: 101-107

23. Sima AAF, Zhang W-X, Cherian PV, Chakrabarti S (1992) Impaired visual evoked potentials and primary axonopathy of the optic nerve in the diabetic BB/W-rat. Diabetologia 35: 602607

24. Sredy J, Sawicki DR, Notrest RR (1991) Polyol pathway activity in nervous tissues of diabetic and galactose-fed rats: effect of dietary galactose withdrawal or tolrestat intervention therapy. $\mathbf{J}$ Diab Complications 5: 42-47
25. Sima AAF, Brown MB, Prashar A et al. (1992) The reproducibility and sensitivity of sural nerve morphometry in the assessment of diabetic peripheral polyneuropathy. Diabetologia 35: $560-569$

26. Creutzfeld OD, Kuhnt V (1973) Electrophysiology and topographical distribution of visual evoked potentials in animals. In: Jung R (ed) Handbook of sensory physiology, VolVII. Springer Verlag, Berlin, pp 595-646

27. Sima AAF, Nathaniel V, Bril V, McEwen TAJ, Greene DA (1988) Histopathological heterogeneity of neuropathy in insulindependent and non-insulin-dependent diabetes, and demonstration of axo-glial dysjunction in human diabetic neuropathy. J Clin Invest 81: $349-364$

28. Greene DA, Lattimer SA, Sima AAF (1988) Perspectives in diabetes. Are disturbances of sorbitol, phosphoinositide and $(\mathrm{Na}, \mathrm{K})$-ATP-ase regulation involved in the pathogenesis of diabetic neuropathy? Diabetes 37:688-693

29. Sima AAF, Brismar T (1985) Reversible diabetic nerve dysfunction. Structural correlates to electrophysiological abnormalities. Ann Neurol 18:21-29

30. Brismar T, Sima AAF, Greene DA (1987) Reversible and irreversible nodal dysfunction in diabetic neuropathy. Ann Neurol 21: 504-507

31. Medori R, Autilio-Gambetti L, Jenich H, Gambetti P (1988) Changes in axonal size and slow axonal transport are related in experimental diabetic neuropathy. Neurology 38: 597-601

32. Tomlinson DR, Townsend J, Fretten P (1985) Prevention of defective axonal transport in streptozocin-diabetic rats by treatment with "Statil" (ICI 128436), an aldose reductase inhibitor. Diabetes 34: 970-972

33. Greene DA, Lattimer SA, Sima AAF (1989) Pathogenesis of diabetic neuropathy: role of altered phosphoinositide metabolism. Crit Rev Neurobiol 5:143-219

34 Sima AAF (1974) Relation between the number of myelin lamellae and axon circumference in fibres of ventral and dorsal roots and optic nerve in normal, undernourished and rehabilitated rats. Acta Physiol Scand 410 [Suppl]: 5-38

35. Wiggins RC, Fuller GN, Dafny N (1982) Propagation of photic evoked responses recorded from the retina, optic chiasm, lateral geniculate body, and visual cortex of the nutritionally rehabilitated rat visual system. Exp Neurol 77: 644-653

36. Puthuraya KP, Nayar U, Deo MG, Manchanda SK (1980) Effects of undernutrition on the visually evoked responses in rats during development. Dev Neurosci 3: 162-173

Received: 22 December 1992

and in revised form: 4 May 1993

Prof. A. A. F. Sima

University of Michigan

1331 E. Ann Street, Box 0580

Ann Arbor, MI 48109

USA 